\title{
Post-disturbance transient stability assessment of power systems towards optimal accuracy-speed tradeoff
}

\author{
Chao Ren ${ }^{1 *}$, Yan $\mathrm{Xu}^{2}$ and Yuchen Zhang ${ }^{3}$
}

\begin{abstract}
The recent development of phasor measurement technique opens the way for real-time post-disturbance transient stability assessment (TSA). Following a disturbance, since the transient instability can occur very fast, there is an urgent need for fast TSA with sufficient accuracy. This paper first identifies the tradeoff relationship between the accuracy and speed in post-disturbance TSA, and then proposes an optimal self-adaptive TSA method to optimally balance such tradeoff. It uses ensemble learning and credible decision-making rule to progressively predict the post-disturbance transient stability status, and models a multi-objective optimization problem to search for the optimal balance between TSA accuracy and speed. With such optimally balanced TSA performance, the TSA decision can be made as fast as possible while maintaining an acceptable level of accuracy. The proposed method is tested on New England 10-machine 39-bus system, and the simulation results verify its high efficacy.
\end{abstract}

Keywords: Ensemble learning, Extreme learning machine (ELM), Intelligent system (IS), Multi-objective optimization, Transient stability assessment

\section{Introduction}

Transient stability refers to the ability of the power system to maintain synchronism after being subjected to a severe disturbance, such as a short circuit on a transmission line [1]. Loss of transient stability can lead to catastrophic events, such as cascading failure and/or wide-spread blackout. Therefore, maintaining transient stability is an essential requirement in power system operation.

Post-disturbance transient stability assessment (TSA) is of great importance to avoid the instability propagation following a disturbance. It predicts the system stability status under an ongoing disturbance, and its assessment decision is utilized to trigger emergency control actions such as generator tripping and/or load shedding. The traditional TSA method is time-domain (T-D) simulation, which iteratively solves a set of high-dimensional non-linear differential algebraic equations [2]. The T-D

\footnotetext{
* Correspondence: renc0003@e.ntu.edu.sg

${ }^{1}$ Interdisciplinary Graduate School, Nanyang Technological University, 50

Nanyang Avenue, Singapore 639798, Singapore

Full list of author information is available at the end of the article
}

simulation is computationally burdensome and requires accurate information of system modelling.

With the wide deployment of phasor measurement units (PMU), power system operating condition can be monitored in real-time, which opens the way for real-time post-disturbance TSA. Based on the real-time system data, a number of direct methods have been proposed to speed up the T-D simulation for post-disturbance TSA [3]. Some examples are piecewise constant-current load equivalent [4], emergency single machine equivalent [5], and post-disturbance trajectory analysis [6]. Although these methods reduce the complexity of the TSA problem, they can only provide conservative and approximate assessment result, and their decision-making speed is still insufficient to timely trigger emergency control actions.

To achieve fast real-time TSA, intelligent system IS-based methods have been identified as a promising solution [7-16]. In IS-based TSA, the conventional T-D simulation data constructs the database to train the intelligent models at offline stage, and then the trained models can perform fast online TSA with the minimal computation effort. In the literature, the intelligent 
models, such as decision tree (DT) [10-12], artificial neural network (ANN) [13] and support vector machine (SVM) [14], have demonstrated their strengths in power system stability assessment.

In most of existing methods, the TSA decision tends to be made at a fixed time following the disturbance. A problem of such TSA implementation is it requires long response time to allow for sufficient TSA accuracy, which can lead to late and ineffective emergency control actions. To improve the TSA response speed, a self-adaptive TSA scheme has been proposed in $[17,18]$. It monitors the credibility of the IS output over a progressively increasing observation window, and delivers the TSA decision once a credible result is obtained. In doing so, the TSA response time can be shortened without the impairment on TSA accuracy. Moreover, it is implied that, under the self-adaptive TSA scheme, the overall TSA accuracy and speed are sensitive to the values of a number of user-defined parameters involved in the credibility monitoring process. However, in $[17,18]$, those parameters are manually tuned, which is time consuming and cannot ensure the optimal TSA performance.

Considering above inadequacies, the contribution of this paper is to first identify the tradeoff relationship between TSA accuracy and speed, and then propose an optimal self-adaptive method that is able to optimally balance the post-disturbance TSA accuracy and speed. In the proposed method, a randomized learning algorithm, extreme learning machine, is adopted owing to its stochastic nature and fast learning capability [15]. Following a disturbance, the transient stability status of the system is progressively predicted by ELM ensemble models, and the credibility of the prediction results is identified through a credible decision-making rule. Moreover, a multi-objective optimization problem (MOP) is modelled to optimally balance the tradeoff between TSA accuracy and speed. With such optimally balanced TSA performance, the TSA decision can be delivered as fast as possible while maintaining an acceptable level of accuracy, so the emergency control actions can be timely and accurately triggered to avoid further blackout events.

The proposed method has been tested on New England 39-bus system, and the simulation results demonstrate accurate and fast post-disturbance TSA.

\section{Problem identification}

With the increasing deployment of PMUs in modern power systems, post-disturbance real-time TSA is of great significance to avoid blackout events. For a successful TSA scheme, the assessment decision should be sufficient accurate and delivered as fast as possible following the disturbance. However, there is an intractable tradeoff problem between TSA accuracy and speed, which will be raised in this section.

\subsection{IS-based post-disturbance TSA}

Transient stability refers to the system's ability to maintain its synchronism subject to a disturbance. It depends on both the initial operating state of the system and the severity of the disturbance [1]. Instability is usually in the form of aperiodic angular separation due to insufficient synchronizing torque. The timeframe for post-disturbance transient stability study is usually 3 to $5 \mathrm{~s}$ after the disturbance [3].

With the development of PMU, post-disturbance TSA can be performed in real-time and in a response-based manner [19]. Based on the massive amount of data from PMUs, IS-based methods have been identified as powerful tools for real-time TSA given the high complexity of the system and the difficulties in modelling the physics behind the complex system dynamics. Compared to traditional TSA methods, such as T-D simulation and direct methods [4-6], the advantages of IS-based methods include their real-time computational speed, less data requirement, strong generalization ability, and versatility [20].

In IS-based methods, the intelligent models need to be trained at the offline stage in advance. The training data is either obtained from historical operating record or generated using T-D simulations on different contingencies. The input and output of an intelligent system for post-disturbance TSA are shown in Fig. 1. The inputs to the IS are the post-disturbance time series of different electrical variables, such as bus voltages, line current, rotor angle of synchronous machines, etc. Based on such multivariate time series input, the IS should be able to predict transient stability status as the TSA result.

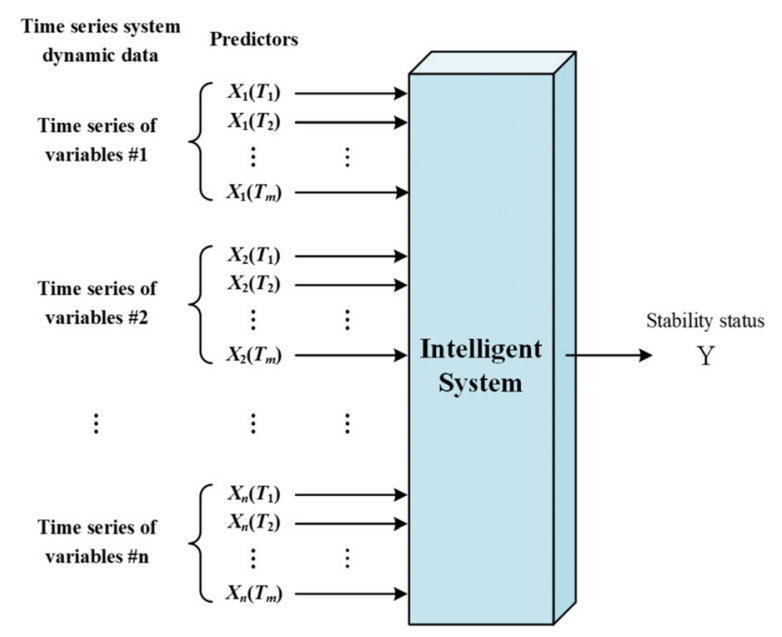

Fig. 1 Illustration of an IS for Post-Disturbance TSA 


\subsection{The self-adaptive TSA scheme}

In the literature, most existing TSA methods utilize a fixed-length observation window and the response time is constant. However, this static time response can be less reliable to cope with fast transient instabilities situation. Moreover, different system models may require substantially different lengths of observation windows to obtain reliable assessment results. In [17, 21, 22], a self-adaptive TSA scheme is proposed and adopted to obtain a reliable assessment result as fast as possible. In doing so, emergency control actions can be activated at an early time to timely avoid further instability propagation.

The structure of the self-adaptive TSA scheme is shown in Fig. 2. There are a series of intelligent models, and each of them operates at a different decision cycle $T_{i}$. Moreover, by using the credibility check, the stability status of the system is predicted progressively after the fault clearance: at each decision cycle $T_{i}$, if the output from an intelligent model is identified as credible, the TSA result will be directly obtained; otherwise, the assessment will continue at the decision cycle $T_{i+1}$.

In above self-adaptive process, a maximum allowable decision-making time should be defined in order to keep the whole system more reliable and activate the emergency control timely. Compared with the existing fixed-time decision-making models, the self-adaptive TSA scheme can deliver the TSA result as fast as possible without impairing the assessment accuracy. In this way, the unnecessary waiting time can be eliminated, hence more time is spared to make emergency control decisions.

\subsection{The tradeoff problem}

In credibility check, the credible criterion is generally defined by a number of credible decision parameters, and a limit of the exiting methods $[17,21,22]$ is that those parameters are usually manually set by experience.
The parameters selection in this way would have a bad impact on the final accuracy, efficiency, and robustness.

Under the self-adaptive TSA scheme, the final TSA performance is sensitive to the value of those credible decision parameters. If the credible criterion defined by those parameters is too loose, most of the outputs from intelligent models will be regarded as credible, then the TSA response speed will be faster. However, due to the loose credible criterion, the credible intelligent model outputs come with lower accuracy, which can lead to unacceptably low TSA accuracy. On the other hand, if the credible criterion is too strict, although the TSA accuracy can be extremely high, only a very small portion of intelligent model outputs are identified as credible at each decision cycle, which leads to slower TSA response. Obviously, there is a tradeoff relationship between TSA accuracy and speed, which is the focus of this paper.

In general, with longer response time, the IS can take advantage of more system dynamic data, so the TSA results tend to be more accurate. However, TSA instability usually occurs very fast, if the response time is too long, the emergency control of the entire power system cannot be started in time, so that system instability cannot be avoided. To tackle such tradeoff problem, this paper proposes an optimal self-adaptive TSA method which can optimally balance the tradeoff between TSA accuracy and speed, so the overall TSA performance is optimized.

\section{Methods}

\subsection{Proposed method}

In the proposed method, ELM-based ensemble model is used as the intelligent model to provide diversified stability prediction outputs, and the credible decision-making rule in [9] is employed as the credibility check mechanism. This section introduces the existing methodologies used in the proposed method.

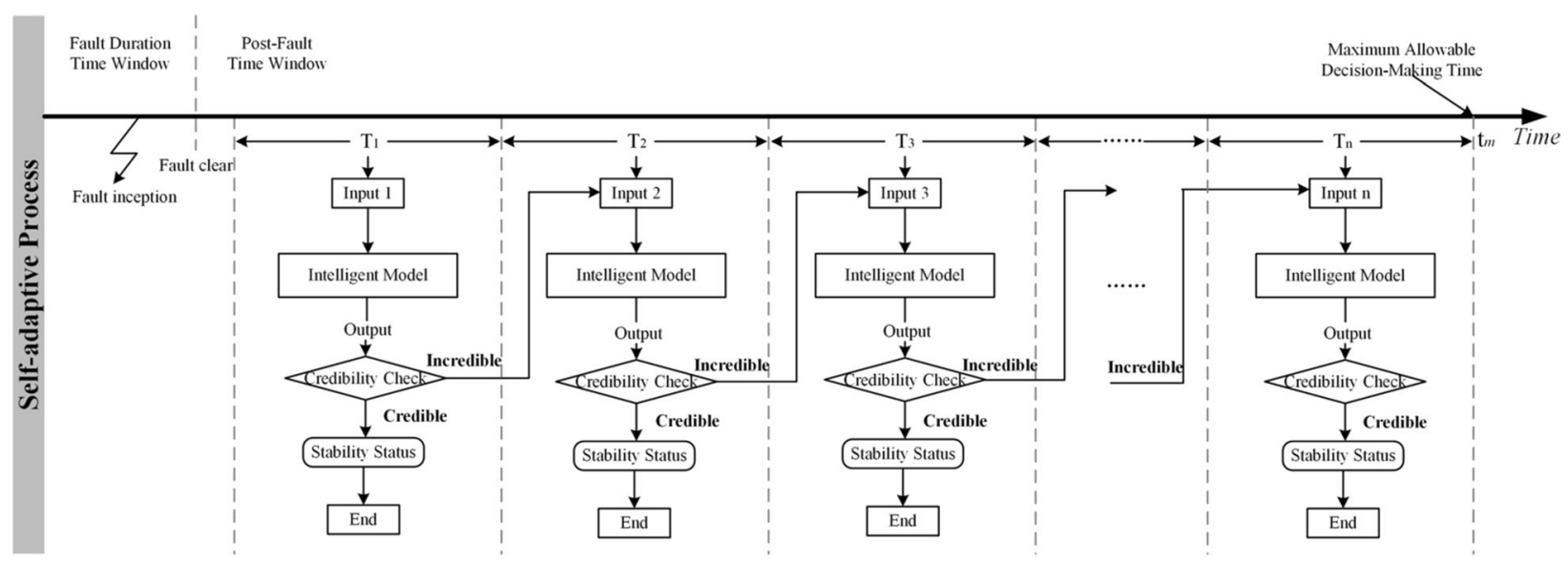

Fig. 2 Structure of the Self-Adaptive TSA Scheme 


\subsection{Extreme learning machine}

ELM is proposed by Huang [15] and receives substantial attention from academic research and practical application. ELM belongs to single-hidden layer feedforward networks (SLFN), and its structure is shown in Fig. 3. ELM includes three layers: input layer, hidden layer, and output layer.

For a standard ELM with $N$ hidden layer nodes, the output function can be mathematically modelled as follows [15]:

$$
\begin{aligned}
f_{\tilde{N}}\left(x_{j}\right) & =\sum_{i=1}^{\tilde{N}} \beta_{i} \cdot g\left(\mathbf{w}_{i} \cdot \mathbf{x}_{j}+b_{i}\right)=t_{j}, \\
j & =1,2, \ldots, N
\end{aligned}
$$

where $g$ represents the activation function, $\mathbf{w}_{i} \in \mathbf{R}^{N}$ is the input weight vector connecting all input layer nodes with the $i$ th hidden layer node, $\beta_{i} \in \mathbf{R}^{N}$ is the output weight vector connecting the $i t h$ hidden layer node with the output layer nodes and $b_{i}$ represents the bias at $i t h$ hidden layer node, $\mathbf{w}_{i} \cdot \mathbf{x}_{j}$ denotes the inner product of $\mathbf{w}_{i}$ and $\mathbf{x}_{j}$.

ELM is completely different from the traditional iterative learning based ANN because the input weights and deviations of ELM are randomly selected, so it can skip the traditional iterative training process, such as back propagation. After that, the output weight $\beta$ is obtained through the analysis of the direct matrix calculation. When the number of hidden nodes is less than the number of training instances, it can be transformed as a linear system for fixed $\mathbf{w}_{i}$ and $b_{i}$, and output weight vector $\boldsymbol{\beta}^{*}$ can be estimated by using the minimal norm least square method as follows:

$$
\boldsymbol{\beta}^{*}=\mathbf{H}^{+} \mathbf{T}
$$

where

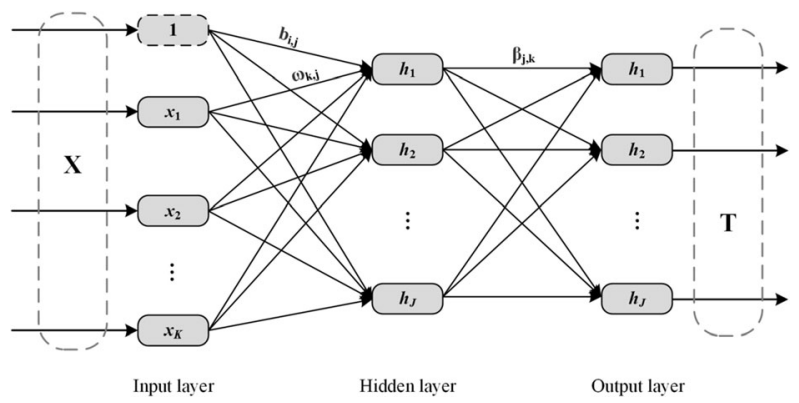

Fig. 3 The Structure of an ELM

$$
\begin{aligned}
\mathbf{H} & =\left[\begin{array}{c}
g\left(x_{1}\right) \\
\vdots \\
g\left(x_{N}\right)
\end{array}\right] \\
& =\left[\begin{array}{ccc}
g\left(\mathbf{w}_{1} \cdot \mathbf{x}_{1}+b_{1}\right) & \cdots & g\left(\mathbf{w}_{\tilde{N}} \cdot \mathbf{x}_{1}+b_{\tilde{N}}\right) \\
\vdots & \ddots & \vdots \\
g\left(\mathbf{w}_{1} \cdot \mathbf{x}_{N}+b_{1}\right) & \cdots & g\left(\mathbf{w}_{\tilde{N}} \cdot \mathbf{x}_{N}+b_{\tilde{N}}\right)
\end{array}\right]
\end{aligned}
$$

In Eq. (2)-(3), $\mathbf{H}$ is called the hidden layer output matrix, and $\mathbf{H}^{\dagger}$ represents the Moor-Penrose generalized inverse of $\mathbf{H}, \mathbf{H}^{\dagger}=\mathbf{H}^{\mathbf{T}}\left(\mathbf{H H}^{\mathbf{T}}\right)^{-1}$.

Compared to the traditional learning algorithms, ELM shows much faster learning speed and only requires much less computation memory for either categorical classification or numeric prediction. Other significant merits of ELM are its efficient tuning mechanism, excellent generalization ability, universal approximation ability, and less parameter adjustment [15]. ELM avoids issues, such as learning rate setting, local minima, and stopping criteria, which are commonly encountered on the traditional learning algorithms. Meanwhile, ELM retains high computation accuracy on many benchmark problems $[9,15,16]$.

\subsection{ELM ensemble learning}

Ensemble learning is the technique of combining multiple learning units to solve the same classification or regression problem. In the literature, ensemble learning has been widely used in power system dynamic security assessment $[9,10,12,16,23]$. For ensemble learning, a set of single learning units are individually trained and combined together to make the final decision. Under such paradigm, the single learning units in the ensemble can compensate each other. This method creates the learning diversity among single learning units, so their aggregated output tends to be more accurate and more robust.

Encouraged by the previous results, this paper uses ELM ensemble model to make stability status prediction. Since ELM adopts random input weights and biases, its training speed is significantly improved, so the increased training burden of ensemble training can be greatly alleviated. Moreover, in ensemble training, each single ELM not only selects random input weights and bias, but also randomly selects training data, hidden node number and activation function. By this way, the ELM ensemble generates more diversified outputs for better overall prediction performance. For each single ELM in the proposed ELM ensemble model, the specific training process is as follow:

\section{Single Learning Unit Training}

Given a database of $S \times F$ size to train $E$ single ELMs, where $S$ is the number of instances and $F$ is the number of input features. 
For $i=1$ to $E$ :

1) Randomly sample $s \in[1, S]$ instances in the database.

2) Randomly select $f \in[1, F]$ features in the feature set.

3) Randomly assign an effective activation function $h_{E}$ and the number of hidden layer nodes from the optimal range $\left[h_{M I N}, h_{M A X}\right]$ (Subject to a pre-tuning procedure).

4) Train the ELM by using the selected instances, features, activation function, and number of hidden layer nodes.

\section{End}

The performance of ELM ensemble model on post-fault transient stability assessment has been tested in [17], and the final results verify its excellence in accuracy, robustness, and reliability compared to a single ELM.

\subsection{Credible decision-making rule}

In practical classification, some instances may be very close to the boundary decision of the regression output. It has been shown that the output value of most of the wrong classifications is very close to the mean of the class labels [16]. On the other hand, because of the prediction error, it is necessary to define the specific threshold as the decision boundary for classification [16]. Obviously, the deviation between the actual output and the predefined class label can be obtained from the prediction error. For single classification, each individual ELM classifier exports outputs about whether the classification is credible or not. For a credible classification, it is comprised of classified stable and unstable.

Based on above cases, the credible decision-making rule proposed in [9] is employed to check the credibility of ELM ensemble output. Suppose the ensemble model includes totally $E$ single ELMs, then we define that the predicted outputs of single ELMs are divided into three different classes in order to improve classification reliability. In this paper, for post-disturbance TSA, the stability class labels are represented by binary numeric values, 1 (stable) and -1 (unstable), then the specific credible classification rule of every ELM is as follow:

$$
\text { If }\left\{\begin{array}{c}
y_{i} \in\left[l b_{s}, u b_{s}\right] \Rightarrow y_{i}=+1 \quad \text { (Credible-stable) } \\
y_{i} \in\left[l b_{u}, u b_{u}\right] \Rightarrow y_{i}=-1 \quad(\text { Credible-unstable }) \\
y_{i} \in\left(-\infty, l b_{u}\right) \text { or }\left(u b_{u}, l b_{s}\right) \text { or }\left(u b_{s},+\infty\right) \Rightarrow y_{i}=0 \\
\quad(\text { Incredible })
\end{array}\right.
$$

where $y_{i}$ represents the predicted output of each ELM in the ensemble, $0<l b_{s}<1, u b_{s}>1, l b_{u}<-1,-1<u b_{u}<0$ are the boundaries to respectively distinguish the stable, unstable, and incredible $y_{i}$.
Due to the lack of knowledge of the available training data, the fitting distribution may not always be realistic. This can be the core reason accounting for the prediction error on unknown instances. The estimation of the credibility of the ensemble outputs is based on the portion of single ELM outputs that are recognized incredible using (4). A larger portion of incredible ELM outputs generally means the ensemble output is less reliable.

\section{Credible Decision-Making Rule}

Given totally $E$ single ELMs, which can totally obtain $s$ "0" outputs, $u$ “ +1 " outputs and $v "-1$ " outputs $(s+u+v=E)$.

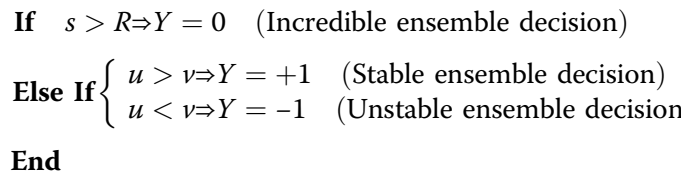

In (5), $Y$ is the ultimate classification result; $R$ is the threshold that determines whether $Y$ is a credible. In the credible decision-making rule, the boundaries $\left[l b_{w} u b_{w}\right.$ $l b_{s}, u b_{s}$ ], the quantity of ELM $m$, and the threshold $R$ are the credible decision parameters that defines the credible criterion of the ELM ensemble output.

\subsection{Accuracy-speed tradeoff optimization}

As earlier mentioned, there is tradeoff relationship between TSA accuracy and speed, and the overall TSA performance under a self-adaptive scheme is sensitive to the value of the credible decision parameters. In this section, the tradeoff relationship between TSA accuracy and speed is modelled in a MOP, based on which the credible decision parameters can be optimized to achieve the best TSA performance.

\subsection{TSA performance metrics}

Before optimizing the TSA performance, appropriate metrics should be defined to quantify the TSA performance. Under the self-adaptive TSA scheme, the TSA performance is evaluated in a statistical way. TSA accuracy and speed are the two priority terms to describe post-disturbance TSA performance. The TSA accuracy is evaluated using the average TSA accuracy (ATA) metrics while the TSA speed is evaluated using the average response time (ART) metrics. They are defined as follows [17]:

$$
A R T=\frac{\sum_{i=1}^{m}\left[T_{i} \times C\left(T_{i}\right)\right]}{\sum_{i=1}^{m} C\left(T_{i}\right)}
$$




$$
A T A=\frac{1}{m} \cdot \sum_{i=1}^{m}\left[\frac{C(T)-M(T)}{C(T)}\right]
$$

where $m$ represents the total number of decision cycles; $T_{i}$ represents the $i$ th decision cycle; $C\left(T_{i}\right)$ and $C(T)$ are the total number of classified instances 'at' and 'until' the current decision cycle, respectively; $M(T)$ is the total number of misclassified instances at the current decision cycle.

The ART index refers to the average time spent to complete TSA following a disturbance. Shorter ART means higher TSA speed. The ATA index computes the overall TSA accuracy on a set of instances, which can represent the accuracy of the proposed method.

\subsection{The multi-objective optimization problem}

Since there is trade-off between speed (ART) and accuracy (ATA), achieving optimization on one side cannot be global optimal. Thus, the optimization of the credible decision parameters can be formulated in a multi-objective optimization problem as follow:

$$
\begin{aligned}
& \text { Maximize } \quad \mathbf{p}(\mathbf{x})=\left[-p_{1}(\mathbf{x}), p_{2}(\mathbf{x})\right]=[-A R T, A T A] \\
& \text { S.t. }\left\{\begin{array}{l}
\mathbf{x}=\left[\mathbf{l} \mathbf{b}_{\mathbf{u}}, \mathbf{u} \mathbf{u b}_{\mathbf{u}}, \mathbf{l} \mathbf{b}_{\mathbf{s}}, \mathbf{u b} \mathbf{b}_{\mathbf{s}}, \mathbf{R}\right] \\
\mathbf{l b}_{\mathbf{u}}=\left(l b_{u}^{1}, l b_{u}^{2}, l b_{u}^{3}, \ldots, l b_{u}^{T_{\max }}\right) \\
\mathbf{u b}_{\mathbf{u}}=\left(u b_{u}^{1}, u b_{u}^{2}, u b_{u}^{3}, \ldots, u b_{u}^{T_{\max }}\right) \\
\mathbf{l} \mathbf{b}_{\mathbf{s}}=\left(l b_{s}^{1}, l b_{s}^{2}, l b_{s}^{3}, \ldots, l b_{s}^{T_{\max }}\right) \\
\mathbf{u} \mathbf{b}_{\mathbf{s}}=\left(u b_{s}^{1}, u b_{s}^{2}, u b_{s}^{3}, \ldots, u b_{s}^{T_{\max }}\right) \\
\mathbf{R}=\left(R^{1}, R^{2}, R^{3}, \ldots, R^{T_{\max }}\right) \\
l b_{u}^{n}<-1,-1<u b_{u}^{n}<0,0<l b_{s}^{n}<1, u b_{s}^{n}>1 \\
0<R^{n}<200
\end{array}\right.
\end{aligned}
$$

where the speed ART and accuracy ATA are the two objectives which are related to the credible decision parameters through $p_{1}$ and $p_{2}$, respectively. Under the self-adaptive TSA scheme, the number of decision parameters depends on the maximum allowable decision-making time $T_{\max }$. By solving this specific multi-objective optimization problem, decision makers can obtain the corresponding solutions according to their actual needs.

\subsection{Pareto optimality}

Compared with the single-objective optimization problem, when two or more objectives are equally important in the optimization problem, the optimal solution is not generally unique if there is trade-off between the objectives. On the contrary, by providing a set of optimized solutions, decision makers can choose one of the best solutions according to their actual needs.

The tradeoff relationship can be defined by the Pareto optimality theory. The set of all Pareto optimal solutions is called the Pareto set, and the set of all Pareto optimal target vectors is called the Pareto optimal frontier (POF)
[24]. We can use POF to display an interpretable and remarkable pattern showing the tradeoff between ART and ATA.

\subsection{Model design}

Under self-adaptive TSA scheme, the credible criterion defined for each decision cycle would determine the overall speed and accuracy. This paper proposes an optimal self-adaptive TSA method to optimize the credible decision parameters at different decision cycle for the best overall TSA performance. The proposed method is illustrated in Fig. 4. It is implemented via offline training, performance optimization, and online assessment. The offline training and the performance optimization are preparation works at offline stage, and the online assessment shows how the proposed method performs post-disturbance TSA at online stage.

\subsection{Offline training}

Combining ELM ensemble model, credibility check, and multi-objective optimization, the proposed method has to be prepared at offline stage as shown in Fig. 4. Since the multi-objective optimization is formulated based on the reliable classification performance of ELM ensemble, so the validation process is designed to derive the POF. Owing to self-adaptive mechanism, all of the classification boundaries, credible threshold at each time should be integral optimization. Finally, the trained ELM ensemble, the POF, and the Pareto set can form the reliable ensemble model for online assessment.

\subsection{Performance optimization}

In performance optimization, the cross validation outputs construct an output set, based on which the MOP is solved to search for the optimal TSA performance. Genetic algorithm is used in this paper to solve the MOP, and a POF is generated as the optimization result. The Pareto points in POF should form an interpretable and remarkable pattern showing the tradeoff between TSA accuracy and speed. Since POF includes multiple Pareto points with equal optimality, a compromise solution needs to be selected among them to represent the best TSA performance. Such compromise solution is generally decided based on the practical system's operation need and the operator's experience.

\subsection{Online assessment}

At online stage, the proposed method is triggered when there is a physical fault occurring in the system. Following the fault, the transient stability of the system is assessed in a self-adaptive way based on the progressively collected PMU measurement. A new decision cycle starts every time a new PMU measurement is obtained. The ELM ensemble model predicts the transient 


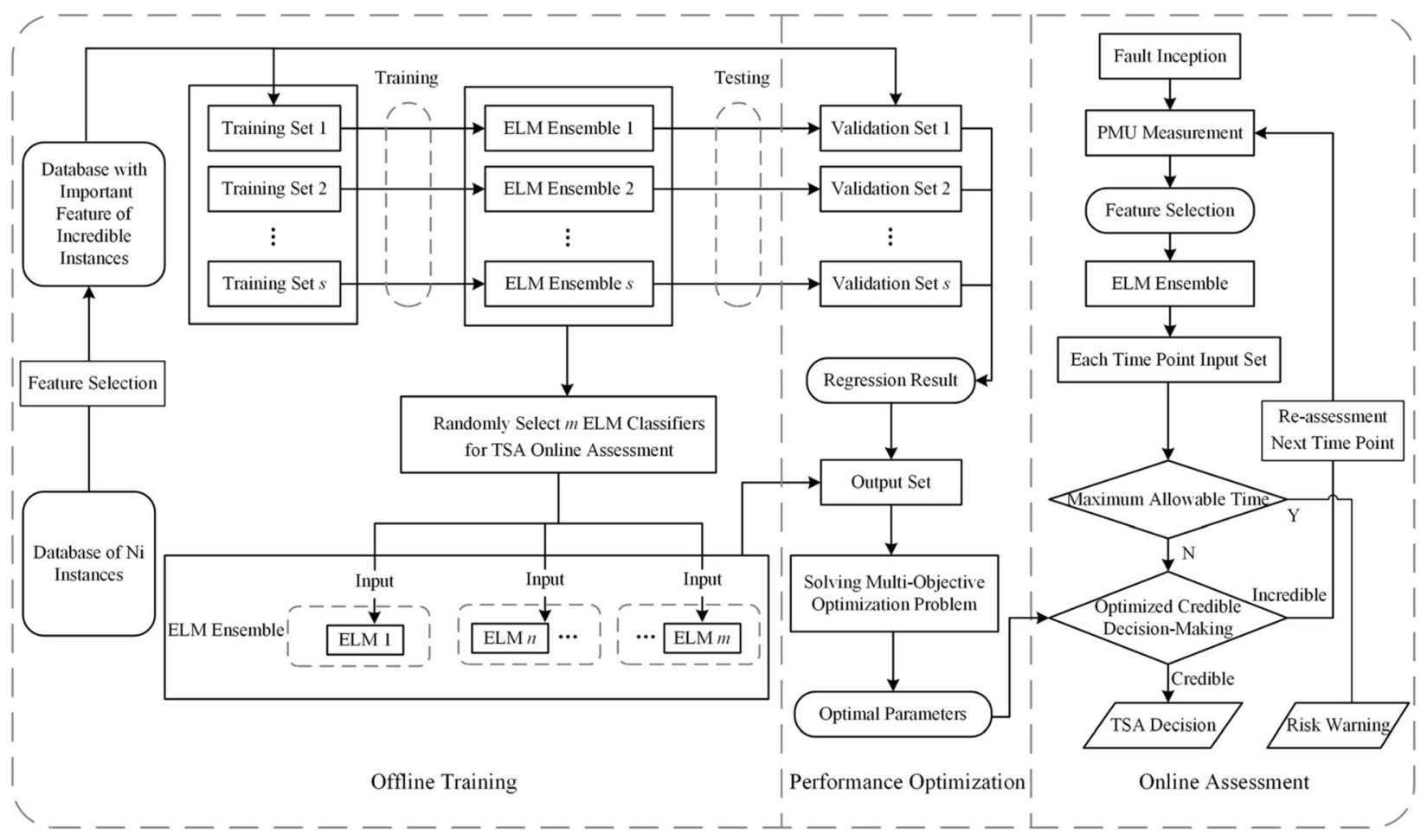

Fig. 4 The Optimal Self-Adaptive TSA Method

stability status at each decision cycle, and the optimal credible decision parameters are used to check the credibility of the ensemble output. If incredible outputs are obtained, the transient stability should be re-assessed at the next decision cycle. Above process continues until a credible TSA decision is obtained or the maximum decision-making time is reached. The online assessment procedure is shown in Fig. 4.

\section{Results and discussion}

\subsection{Numerical test}

The proposed method is tested on New England 10-machine 39-bus system (Fig. 5), which represents a benchmark power system for stability analysis [21]. The synchronous generator at bus 37 is replaced by a wind farm of the same capacity to simulate the impact of renewable energy sources. The simulation and computation in the test is conducted on a 64-bit computer with an Intel Core i7 $\mathrm{CPU}$ working at $2.8-\mathrm{GHz}$ and $16-\mathrm{GB}$ RAM. T-D simulation is performed by using commercial software PSS/E.

\subsection{Database generation}

To have a comprehensive database for post-disturbance TSA, a variety of physical faults are simulated on a wide range of operating scenarios to obtain the post-disturbance system information.

In the test, 6000 operating scenarios are generated by randomly varying the load demand and the generated wind power at bus 37 . The load demand at each load bus varies between 0.8 and 1.2 of its rated values, and the generated wind power at bus 37 varies between 0 and its capacity. Optimal power flow is run to calculate the optimal operating point of each scenario.

For each generated operating scenario, three phase faults with randomly selected fault location and fault clearing time are applied. Considering the practical

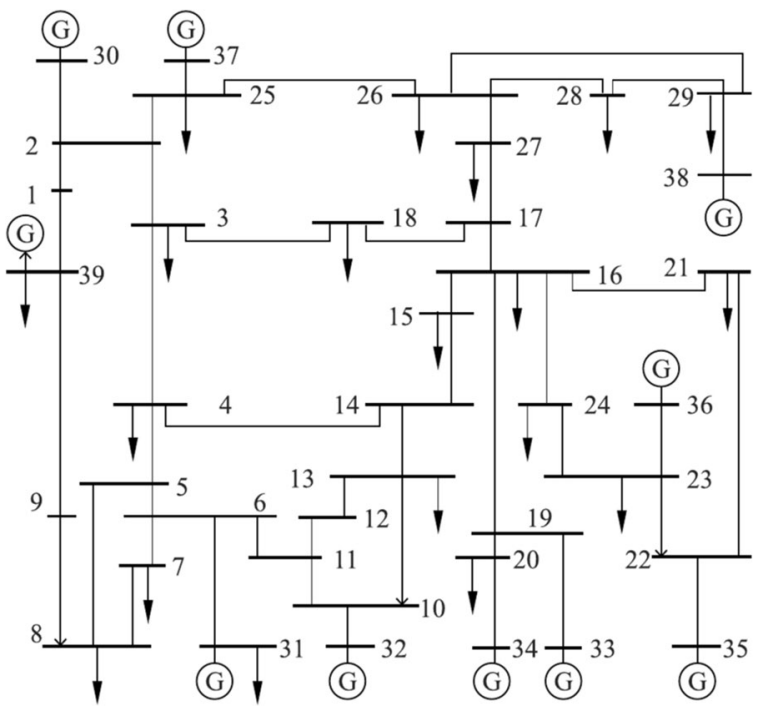

Fig. 5 New England 10-machine 39-bus system 
situation, the fault clearing time is randomly selected between 0.1 and $0.3 \mathrm{~s}$ after the fault inception.

T-D simulation is performed to simulate the dynamics of the system, where the simulation time is $5 \mathrm{~s}$ and the simulation time step is $0.02 \mathrm{~s}$. The voltage and rotor-angle trajectories of synchronous generators are recorded. The generator voltage trajectories are regarded as the input to the intelligent models and the rotor angles are utilized to calculate the transient stability index (TSI) as follow:

$$
T S I=\frac{360-\left|\Delta \delta_{\max }\right|}{360+\left|\Delta \delta_{\max }\right|} \times 100
$$

where $\Delta \delta_{\max }$ is the maximum angle deviation between any two generators at any time point. The TSA status $y$ can be obtained by using TSI as follow:

$$
y_{t}=\left\{\begin{array}{ll}
1 & (\text { Stable) for } T S I>0 \\
0 & \text { (Unstable) for } T S I \leq 0
\end{array} \quad, t=1,2, \ldots, T\right.
$$

As a consequence, the database consists of 4000 instances, each with the post-disturbance generator voltage trajectories as input and the transient stability status as output. The 4000 instances are then randomly divided into two different sets, one serves as training set, whereas the other as testing set. The training set occupies $87.5 \%$ and the testing set occupies $12.5 \%$ of all the instances.

\subsection{ELM ensemble training}

Based on the simulation time step, each decision cycle for the self-adaptive TSA scheme should be $0.02 \mathrm{~s}$. The maximum allowable decision-making time is set at $0.4 \mathrm{~s}$ (i.e. $T_{\max }=20$ ) to keep the whole system more reliable and activate the emergency control timely. Since one ELM ensemble model is trained for each decision cycle, 20 ELM ensemble models are needed to implement self-adaptive TSA. To train each ELM ensemble model, the following parameters need to be specified.

1) Total Number of ELMs in an Ensemble E: As verified by the existing ensemble learning methods [22, 25], with increasing number of single learning units, the overall prediction error will gradually decrease but converge to a limit. In our case, $\mathrm{E}$ is 200.

2) Activation Function and Optimal Hidden Node Range: The number of hidden layer nodes and the choice of activation functions also need to be adjusted in the training process of each single learning unit. For an activation function, the ELM computation accuracy can only be maximized within a specific hidden node range. In the test, the Sigmoid and Sine functions are chosen as the candidate activation functions, and the optimal hidden node range for those two functions is [150, 250].

3) Number of Training Instances: The quantity of instances which are selected to train ELMs determines the performance of ELM ensemble model. In the test, the number of training instances for each single ELM is chosen to be 3500 .

\subsection{Performance optimization result}

In performance optimization, the multi-objective problem is solved by genetic algorithm and the corresponding Pareto solutions are obtained. The POF in terms of ART and ATA is shown in Fig. 6.

The POF is a reference to decide the compromise parameters for online application; meanwhile, it serves as a benchmark for optimal post-disturbance TSA performance. Therefore, by utilizing any Pareto point on POF, the online TSA performance (ART and ATA) could closely achieve its validated optimal performance. Some properties of the obtained POF are listed in Table 1.

From Fig. 6 and Table 1, the tradeoff relationship between TSA accuracy and speed can be clearly observed. On the one hand, with all the ensemble outputs being recognized as credible, the self-adaptive process will be instantly completed at the 1 st decision cycle (i.e. extremely fast TSA speed), but the TSA accuracy is only $90.75 \%$. On the other hand, while 100\% TSA accuracy (i.e. ATA) is achieved, the optimal ART is 8.367 cycles (i.e. 0.16734 s).

Among the Pareto points, a compromise solution needs to be selected to represent the optimal TSA performance. In this paper, a practical requirement of $99 \%$ is assumed for ATA, which regulates the post-disturbance TSA accuracy. Based on such requirement, the Pareto point listed in Table 2 is used as the compromise solution because it satisfies the 99\% ATA requirement with the lowest $\mathrm{ART}$.

In practice, the choice of the compromise solution is not limited to above strategy. During online application, system operators should employ their own decision-making strategy to select the best Pareto point, and they are also able to adjust the choices depending

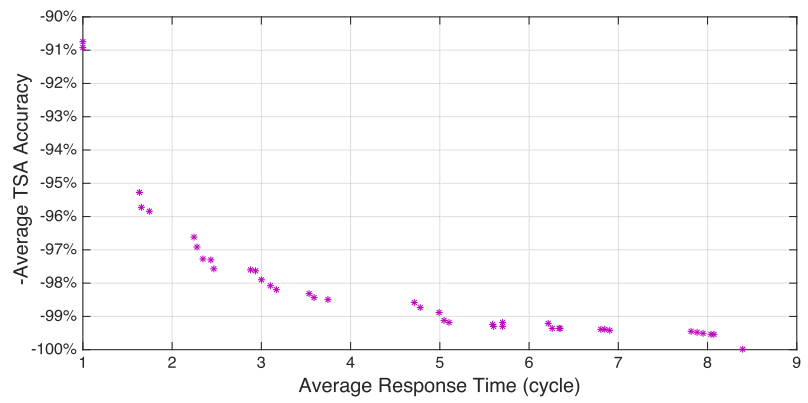

Fig. 6 The POF obtained in Performance Optimization 
Table 1 POF solution results

\begin{tabular}{lll}
\hline No. of Pareto Solutions & Worst Case ART & Worst Case ATA \\
\hline 45 & 8.367 cycles (0.16734 s) & $90.75 \%$ \\
\hline
\end{tabular}

on the practical TSA requirement of the power system. Therefore, the proposed model offers the system operators more flexibility in manipulating the post-disturbance TSA performance.

\subsection{Online testing result}

The proposed method is applied to the testing instances to test its online TSA performance. Besides ART and ATA, the TSA performance at each decision cycle also needs to be investigated. The testing result is shown in Table 3. The columns of the Table 3, from left to right, are respectively the decision cycle, the number of remaining instances to be assessed at each decision cycle, the number of assessed instances at each decision cycle, the assessment accuracy at each decision cycle and the accumulated assessment accuracy up to each decision cycle.

In Table 3, it can be observed that the accuracy at most decision cycles are $100 \%$, which means that the TSA accuracy will not be degraded by the early assessment of some instances. This result verifies that the proposed method can improve the TSA speed while maintaining the TSA accuracy. Moreover, compared to the performance indicated by the selected Pareto point in Table 2, the ATA in the testing result is slightly lower, but the ART is significantly reduced, indicating much faster TSA speed. This testing result further verifies the tradeoff relationship between TSA accuracy and speed.

\section{Conclusion}

This paper focuses on improving post-disturbance TSA performance using IS-based methods. It first identifies a tradeoff problem between TSA accuracy and speed, and then proposes an optimal self-adaptive TSA method to optimally balance such tradeoff and thereby achieve the best overall TSA performance. The proposed method adopts ELM algorithm and ensemble learning techniques to predict transient stability status at each decision cycle, and uses a credible decision-making rule to identify the credibility of the ELM ensemble output. The post-disturbance TSA is performed under a self-adaptive scheme for gain fast assessment ability, so the emergency control actions can be activated in time to prevent the power system against catastrophic blackout. Under a

Table 2 Selected pareto point

\begin{tabular}{ll}
\hline Average Response Time (ART) & Average TSA Accuracy (ATA) \\
\hline 5.536 cycles (0.11072 s) & $99.27 \%$ \\
\hline
\end{tabular}

Table 3 Testing results

\begin{tabular}{|c|c|c|c|c|}
\hline $\begin{array}{l}\text { Decision } \\
\text { Cycles } \\
\end{array}$ & $\begin{array}{l}\text { No. of Remained } \\
\text { Instances }\end{array}$ & $\begin{array}{l}\text { No. of Assessed } \\
\text { Instances }\end{array}$ & $\begin{array}{l}\text { Each Cycle } \\
\text { Accuracy }\end{array}$ & $\begin{array}{l}\text { Total } \\
\text { Accuracy } \\
\end{array}$ \\
\hline 1 (0.02 s) & 500 & 164 & $99.39 \%$ & $99.39 \%$ \\
\hline $2(0.04 \mathrm{~s})$ & 336 & 24 & $100 \%$ & $99.47 \%$ \\
\hline 3 (0.06 s) & 312 & 47 & $100 \%$ & $99.57 \%$ \\
\hline $4(0.08 \mathrm{~s})$ & 265 & 58 & $100 \%$ & $99.66 \%$ \\
\hline 5 (0.10s) & 207 & 11 & $100 \%$ & $99.67 \%$ \\
\hline $6(0.12 \mathrm{~s})$ & 196 & 33 & $100 \%$ & $99.70 \%$ \\
\hline 7 (0.14 s) & 163 & 7 & $71.43 \%$ & $99.13 \%$ \\
\hline $8(0.16 \mathrm{~s})$ & 156 & 12 & $91.67 \%$ & $98.88 \%$ \\
\hline 9 (0.18 s) & 144 & 0 & $\mathrm{~N} / \mathrm{A}$ & $98.88 \%$ \\
\hline 10(0.20s) & 144 & 1 & $100 \%$ & $98.88 \%$ \\
\hline $11(0.22 \mathrm{~s})$ & 143 & 10 & $90.00 \%$ & $98.64 \%$ \\
\hline $12(0.24 \mathrm{~s})$ & 133 & 0 & N/A & $98.64 \%$ \\
\hline $13(0.26 s)$ & 133 & 3 & $100 \%$ & $98.65 \%$ \\
\hline $14(0.28 \mathrm{~s})$ & 130 & 1 & $100 \%$ & $98.65 \%$ \\
\hline 15(0.30s) & 129 & 1 & $100 \%$ & $98.66 \%$ \\
\hline $16(0.32 \mathrm{~s})$ & 128 & 5 & $100 \%$ & $98.67 \%$ \\
\hline $17(0.34 \mathrm{~s})$ & 123 & 0 & $\mathrm{~N} / \mathrm{A}$ & $98.67 \%$ \\
\hline $18(0.36 \mathrm{~s})$ & 123 & 0 & $\mathrm{~N} / \mathrm{A}$ & $98.67 \%$ \\
\hline $19(0.38 \mathrm{~s})$ & 123 & 3 & $100 \%$ & $98.68 \%$ \\
\hline $20(0.40 s)$ & 120 & 3 & $100 \%$ & $98.69 \%$ \\
\hline ART & \multicolumn{2}{|c|}{3.572 cycles ( $0.07144 \mathrm{~s})$} & ATA & $99.01 \%$ \\
\hline
\end{tabular}

multi-objective optimization framework, the parameters to define the credible decision-making rule are optimized and the trade-off between TSA accuracy and speed is also optimally balanced. Moreover, the proposed method also enables system operators to empirically select their compromise TSA performance from the obtained POF. The proposed TSA method has been tested on New England 10-machine 39-bus system, and the simulation results verifies the tradeoff relationship between TSA accuracy and speed and demonstrates high TSA performance of the proposed method.

\section{Abbreviations \\ ANN: Artificial neural network; ART: Average response time; ATA: Average TSA accuracy; DT: Decision tree; ELM: Extreme learning machine; IS: Intelligent system; MOP: Multi-objective optimization problem; PMU: Phasor measurement unit; POF: Pareto optimal frontier; SLFN: Single- hidden layer feedforward networks; SVM: Support vector machine; T-D: Time- domain; TSA: Transient stability assessment; TSI: Transient stability index}

Availability of data and materials

Data sharing not applicable to this article as no datasets were generated or analyzed during the current study. Please contact author for data requests.

Authors' contributions

CR conceived and designed the study. $C R$ and $Y X$ performed the experiments and analyzed. $Y Z$ generated the needed database. CR, YX and 
YZ wrote the paper, reviewed and edited the manuscript. All authors read and approve the manuscript.

\section{Competing interests}

The authors declare that they have no competing interests.

\section{Author details}

${ }^{1}$ Interdisciplinary Graduate School, Nanyang Technological University, 50 Nanyang Avenue, Singapore 639798, Singapore. ${ }^{2}$ School of Electrical and Electronic Engineering, Nanyang Technological University, Singapore, Singapore. ${ }^{3}$ School of Electrical Engineering and Telecommunication, University of New South Wales, Sydney, Australia.

Received: 2 March 2018 Accepted: 11 May 2018

Published online: 22 June 2018

\section{References}

1. Kundur, P., Paserba, J., Ajjarapu, V., Andersson, G., Bose, A., Canizares, C., Hatziargyriou, N., Hill, D., Stankovic, A., \& Taylor, C. (2004). Definition and classification of power system stability IEEE/CIGRE joint task force on stability terms and definitions. IEEE Trans Power Syst, 19(3), 1387-1401.

2. A.-A. Fouad, and V. Vittal, Power system transient stability analysis using the transient energy function method: Pearson Education, 1991.

3. Chiang, H.-D. (2011). Direct methods for stability analysis of electric power systems: theoretical foundation, BCU methodologies, and applications. John Wiley \& Sons.

4. Liu, C.-W., \& Thorp, J. (1995). Application of synchronised phasor measurements to real-time transient stability prediction. IEE ProceedingsGeneration, Transmission and Distribution, 142(4), 355-360.

5. Pavella, M., Ernst, D., \& Ruiz-Vega, D. (2012). Transient stability of power systems: a unified approach to assessment and control. Springer Science \& Business Media.

6. Gurusinghe, D. R., \& Rajapakse, A. D. (2016). Post-disturbance transient stability status prediction using synchrophasor measurements. IEEE Trans Power Syst, 31(5), 3656-3664.

7. Kundur, N., Balu, J., \& Lauby, M. G. (1994). Power system stability and control. New York: McGraw-hill.

8. Zhou, Z.-H., Wu, J., \& Tang, W. (2002). Ensembling neural networks: Many could be better than all. Artif Intell, 137(1-2), 239-263.

9. Xu, Y., Dong, Z. Y., Zhao, J. H., Zhang, P., \& Wong, K. P. (2012). A reliable intelligent system for real-time dynamic security assessment of power systems. IEEE Trans Power Syst, 27(3), 1253-1263.

10. Kamwa, I., Samantaray, S., \& Joos, G. (2010). Catastrophe predictors from ensemble decision-tree learning of wide-area severity indices. IEEE Transactions on Smart Grid, 1(2), 144-158.

11. Guo, T., \& Milanovic, J. V. The effect of quality and availability of measurement signals on accuracy of on-line prediction of transient stability using decision tree method. In Innovative Smart Grid Technologies Europe (ISGT EUROPE), 2013 4th IEEE/PES (pp. 1-5). IEEE.

12. He, M., Vittal, V., \& Zhang, J. (2013). Online dynamic security assessment with missing pmu measurements: A data mining approach. IEEE Trans Power Syst, 28(2), 1969-1977.

13. Guo, Z., Zhao, W., Lu, H., \& Wang, J. (2012). Multi-step forecasting for wind speed using a modified EMD-based artificial neural network model. Renew Energy, 37(1), 241-249.

14. Moulin, L., Da Silva, A. A., El-Sharkawi, M., \& Marks, R. J. (2004). Support vector machines for transient stability analysis of large-scale power systems. IEEE Trans Power Syst, 19(2), 818-825.

15. Huang, G.-B., Zhu, Q.-Y., \& Siew, C.-K. (2006). Extreme learning machine: Theory and applications. Neurocomputing, 70(1), 489-501.

16. Zhang, Y., Xu, Y., Dong, Z. Y., Xu, Z., \& Wong, K. P. (2017). Intelligent early warning of power system dynamic insecurity risk: Toward optimal accuracy-earliness tradeoff. IEEE Transactions on Industrial Informatics, 13(5), 2544-2554.

17. Zhang, R., Xu, Y., Dong, Z. Y., \& Wong, K. P. (2015). Post-disturbance transient stability assessment of power systems by a self-adaptive intelligent system. IET Generation, Transmission \& Distribution, 9(3), 296-305.

18. James, J., Hill, D. J., Lam, A. Y., Gu, J., \& Li, V. O. (2018). Intelligent timeadaptive transient stability assessment system. IEEE Trans Power Syst, 33(1), 1049-1058.
19. Begovic, M., Novosel, D., Karlsson, D., Henville, C., \& Michel, G. (2005). Widearea protection and emergency control. Proc IEEE, 93(5), 876-891.

20. Xu, Y., Dong, Z., Meng, K., Zhang, R., \& Wong, K. (2011). Real-time transient stability assessment model using extreme learning machine. IET generation, transmission \& distribution, 5(3), 314-322.

21. Cepeda, J. C., Rueda, J. L., Colomé, D. G., \& Erlich, I. (2015). Data-miningbased approach for predicting the power system post-contingency dynamic vulnerability status. International Transactions on Electrical Energy Systems, 25(10), 2515-2546.

22. Kamwa, I., Samantaray, S., \& Joos, G. (2009). Development of rule-based classifiers for rapid stability assessment of wide-area post-disturbance records. IEEE Trans Power Syst, 24(1), 258-270.

23. Dong, Z. Y., Xu, Y., Zhang, P., \& Wong, K. P. (2013). Using IS to assess an electric power system's real-time stability. IEEE Intell Syst Mag, 28(4), 60-66.

24. Deb, K. (2001). Multi-objective optimization using evolutionary algorithms. John Wiley \& Sons.

25. Amjady, N., \& Banihashemi, S. (2010). Transient stability prediction of power systems by a new synchronism status index and hybrid classifier. IET generation, transmission \& distribution, 4(4), 509-518.

\section{Submit your manuscript to a SpringerOpen ${ }^{\circ}$ journal and benefit from:}

- Convenient online submission

- Rigorous peer review

- Open access: articles freely available online

- High visibility within the field

- Retaining the copyright to your article

Submit your next manuscript at $>$ springeropen.com 